\title{
APORTES PARA DESARROLLAR UNA PRÁCTICA PREPROFESIONAL REFLEXIVA EN LAS CARRERAS DE EDUCACIÓN
}

\author{
Herlinda Guevara López \\ Universidad Católica Sedes Sapientiae
}

Fecha de recepción: septiembre de 2012 Fecha de aceptación: octubre de 2012

Resumen: Las prácticas preprofesionales en los alumnos de Ciencias de la Educación requieren una reformulación que trascienda su condición de requisito formal, tradicionalmente, concebido para algunos colegios en la formación de profesores. Se propone que la práctica sea el espacio donde los estudiantes más allá de la demostración de dominio de un conjunto de teorías, procedimientos y recursos de trabajo docente, debe estimular

* Herlinda Guevara López es doctora en Ciencias de la Educación de la Sección de Post Grado de la Facultad de Educación de la Universidad San Martín de Porres. Especialista en Autoevaluación y Acreditación Universitaria desde 2010 por la Asamblea Nacional de Rectores, así como en Docencia y Didáctica Universitaria, Teoría Curricular, Investigación Educativa, Andragogía y Salud Pública, con amplia trayectoria desempeńando labores docentes y académicas en importantes instituciones educativas y universitarias. Ha desarrollado proyectos de innovación pedagógica, sistemas curriculares e investigación educativa.

Es miembro del Colegio de Doctores en Educación desde 1998. Autora de diversos artículos académicos y textos universitarios e investigaciones como Metodología para la determinación del Perfil Socio-Económico y Académico de los Estudiantes de la Facultad de Odontologia de la USMP (2002), Perfil de las Competencias Digitales de los Docentes del Cono Norte de Lima Metropolitana (2010). Recibió el tercer lugar en XX Concurso Nacional de Educación Horacio 2011, organizado por la Derrama Magisterial y auspiciado por Ministerio de Educación, por la presentación del ensayo pedagógico Inclusión de la robótica educativa en el diseño curricular. 
el desarrollo de unan visión reflexiva de sus propias acciones, conducidas a la enseñanza y cuidado real de las demandas de sus futuros estudiantes y la comunidad educativa; proponiendo así, las estrategias de innovación e iniciativas de investigación con la metodología de investigación-acción.

Palabras Clave: práctica preprofesional, docencia, educación, reflexión e investigación-acción

ABSTRACT: The pre-professional practice among students in science education requires a reformulation that transcends its status as a formal requirement traditionally conceived for some colleges of teacher education. It is proposed that the pre-professional practice is the space where the students beyond the demonstration of mastery of a set of theories, procedures and resources of teaching work should stimulate the development of a reflective view of their own actions conducive to teaching the actual care of the demands of their prospective students and the educational community, thus proposing the innovation strategies and research initiatives using the methodology of action research.

KEYwords: pre-professional practice, teaching, education, reflection, action research

L as prácticas preprofesionales permiten a los alumnos-practicantes desarrollar un sentido de realidad que los aproxima a las diferentes problemáticas inherentes a la profesión docente, de manera integrada entre teoría y práctica educativas. Sin embargo, convencionalmente son consideradas como el momento en que se comprueba lo que el estudiante ha aprendido, es decir, la instancia final del proceso de formación docente del estudiante de educación. Más allá de ello, consideramos que constituyen un espacio de integración donde se profundizan los conocimientos y 
capacidades obtenidas durante la formación docente. En consecuencia, los estudiantes ejercen su función docente pedagógica.

Por otro lado, estas deben constituir un eje de aplicación distribuido a lo largo de la carrera, en torno a los objetivos de la formación general, la formación pedagógica y la formación especializada. Dichos aspectos se integran en función del perfil profesional docente. De esta manera la práctica preprofesional debe ser un proceso de aprendizaje y ejercitación sistemática, progresiva y acumulativa en el análisis y la reflexión sobre la práctica escolar, la aplicación de conocimientos y destrezas, la adquisición y perfeccionamiento de las competencias docentes, la identificación y el compromiso vocacional con la profesión.

Las actividades y experiencias propuestas a los alumnos-practicantes durante la práctica profesional deben permitirles tener la oportunidad de probar y demostrar habilidades y destrezas en el análisis y solución de casos reales de la profesión. Así, aprenderán a utilizar con mayor acierto los conocimientos teóricos y prácticos adquiridos, tutelados por docentes experimentados. Este momento de la formación profesional debe constituirse en un espacio donde el alumno-practicante tenga la oportunidad no solo de aplicar lo aprendido, sino de vivir un proceso de investigación-acción, entendida como un camino que hace del futuro maestro un profesional que reflexiona en la acción y sobre la acción. En consecuencia, mejora su práctica y vivencia sus teorías pedagógicas, que conducen a un cambio profundo en las actividades rutinarias del aula y a lograr un aprendizaje activo y eficiente de los alumnos.

Sayago y Chacón (2006:57) señalan que en las prácticas profesionales confluyen distintos factores. Los autores se refieren a concepciones de enseñanza, aprendizaje, modelos y tradiciones de formación docente, modalidades de gestión y administración de instituciones escolares y las 
características propias del contexto socio cultural. Estas abren un abanico de posibilidades al estudiante, quien analiza críticamente su actuación de aprendiz y de docente; a la vez tiene oportunidad de discutir, contrastar y reconstruir la experiencia docente asumiéndola como objeto de análisis. En ese sentido, las prácticas preprofesionales conducen a acciones integradas de efectos desencadenantes, repercutiendo directamente en la conformación de la profesionalidad, apuntan a reafirmar los fines y propósitos establecidos en el currículo.

Por ello, es relevante reflexionar en torno a los objetivos y alcances de las prácticas preprofesionales. ¿Qué se supone que deben aprender nuestros futuros docentes y, en todo caso, para qué y cómo aprenderlo? Recordemos, asimismo, que la complejidad de la práctica preprofesional requiere la «adquisición de conocimientos», destrezas, valores y actitudes, y aprender las características y función social de la profesión.

\section{PERSPECTIVAS DE LA PRÁCTICA PREPROFESIONAL}

Las prácticas preprofesionales en educación como en otras carreras profesionales deberían estar indisolublemente ligadas a todo el proceso de formación profesional, pues integran los objetivos de los diferentes ámbitos de formación propuestos explícitamente en el perfil de la carrera. No obstante, es frecuente que las prácticas preprofesionales se definan con un marco de referencia, que únicamente se queda en el manejo de habilidades y destrezas. Esta condición tiende a perder de vista los vínculos entre los diversos componentes del currículo. La idea predominante, tradicionalmente, es asumir lineamientos pautados y predeterminados hacia el disciplinar en una práctica instrumental, reduciéndola a ser uno de los pocos constructos que se ha mantenido casi inalterable frente a la dinámica social en constantes cambios. 
Siempre que se reflexiona acerca de la formación docente es recurrente insistir en la relación que necesariamente tiene que darse entre la teoría y la práctica. Pero, a pesar de esto, en el sistema universitario de nuestro país son escasas las experiencias formativas que consiguen articular esa relación de manera satisfactoria, contribuyendo sin proponérselo a una inadecuada formación docente. Esto se evidencia en un significativo número de egresados de educación que refieren un desencuentro, durante su formación y su etapa de prácticas preprofesionales, con los modos de vincular, eficientemente, los referentes teóricos con el quehacer en las aulas como docentes.

Este hecho, en gran parte, no es responsabilidad de los alumnospracticantes. Todo apunta a los responsables de planificar las prácticas preprofesionales, debido a la falta de proyección y organización de esta experiencia formativa, sobre todo como resultado de la percepción del propio proceso educativo y del papel que consideran que deben asumir los futuros docentes.

Para tener una aproximación a las diversas perspectivas con las que se aborda la práctica preprofesional consideraremos la división propuesta por Escobar (2007:185-186). La propuesta refleja las concepciones de los docentes supervisores sobre el proceso de formación docente y la interacción teoría-práctica en dicho proceso: perspectiva racionalista, la perspectiva técnica o de mercado social, y la perspectiva reflexiva.

- Perspectiva racionalista. Aquí la práctica preprofesional es entendida como una aplicación consciente de la teoría, orientada a superar la práctica rutinaria y artesanal; desde esta perspectiva, la formación del futuro docente debe apoyarse en una importante fase de formación teórica inicial que dote al alumno-practicante con los conocimientos y teorías que requerirá en su desempeño. Se trata de la formación de un docente individualista, autónomo, intelectual; y se da poca importancia 
al conocimiento pedagógico que no está relacionado con las disciplinas y al conocimiento que se deriva de la "práctica».

- Perspectiva técnica o de mercado social. En esta perspectiva, la formación se concibe como desarrollo de habilidades y competencias técnicas que garantizan una intervención eficaz. Las prácticas preprofesionales, por consiguiente, se conciben como un entrenamiento de habilidades y competencias; se considera como el componente fundamental en el proceso de formación docente.

El conocimiento teórico se limita a un valor técnico o instrumental. A partir de ello, el proceso de formación inicial se considera un proceso de inducción en la práctica, en la que se entrenan las habilidades para una intervención técnica eficaz. El docente, entonces, es un técnico apoyado en el conocimiento elaborado por otros científicos, por lo que no necesita acceder al conocimiento científico; solamente debe dominar rutinas de intervención técnica. Esta racionalidad técnica produce una subordinación del maestro y por ende, del practicante frente a quien produce el conocimiento.

- Perspectiva reflexiva. Aquí se visualiza la formación del docente como el desarrollo de complejas competencias profesionales de pensamiento y de acción, un largo proceso de interacción de la teoría-práctica, del desarrollo de la capacidad de comprensión situacional que favorezca los juicios razonados y decisiones inteligentes y reflexivas, ambiguas y dinámicas de la vida en las aulas. En este caso, la práctica «se apoya en la interpretación de las situaciones particulares como un todo y no pueden mejorarse sino se mejoran dichas interpretaciones».

Se requiere del docente la habilidad intelectual para discernir la respuesta apropiada a una situación compleja, abierta a la duda y a la incertidumbre. El docente como un profesional reflexivo, capaz de 
comprender la realidad en la que intervenga, debe desarrollar y construir esquemas flexibles de pensamiento y actuación, la investigación y la innovación de propuestas alternativas fundamentadas. Un modelo de esta naturaleza implica la superación de perspectivas como la racionalista y la técnica, y que requiere de la comprensión e interpretación de la singularidad de cada situación concreta apoyada en teorías, experiencias previas, pero no limitadas a estas.

Es evidente que las prácticas preprofesionales exceden la demostración y aplicación de conocimientos y técnicas adquiridas por el futuro docente durante su formación profesional. Por ello, es necesario propiciar la autorreflexión y valoración de las acciones pedagógicas ejecutadas por los alumnos-practicantes, de manera que constituyan experiencias estimuladoras hacia el conocimiento de sí mismo y la capacidad para aprender permanentemente en su futuro desempeño como profesional de la educación.

\section{LOS OBJETIVOS DE LA PRÁCTICA PREPROFESIONAL}

En la actualidad la formación docente debe responder a las demandas de una sociedad en constante cambio ante los avances tecnológicos y su influencia en todos los ámbitos desde el sector educativo, económico, político, empresarial, ético y religioso. Así, las prácticas preprofesionales deben integrar el desarrollo de las habilidades propias del desempeño docente en sus diferentes dimensiones, integrándose estas a las capacidades que exige el mercado de trabajo, las necesidades de la propia disciplina que incluyen las competencias profesionales y la investigación.

Sime (2004) señala que en los últimos años se ha ido profundizando un nuevo paradigma de la profesión docente que busca superar la visión tecnocrática de la docencia presente en ciertas tendencias de formación 
inicial y el desarrollo profesional. Esta concibe al docente educador como operador del currículo, de las metodologías definidas y sistematizadas por otros docentes, lo que implicaba una baja en la autoestima, escasa motivación y poca capacidad de repensar el sentido de la profesión. Ante lo cual enfatiza que están formulando nuevas visiones de la acción docente que permiten explorar potencialidades para el docente como «intelectual crítico» $\mathrm{y}$ «docente investigador».

Desde esta perspectiva, las prácticas preprofesionales deben contribuir a valorar y descubrir los problemas y potencialidades de la profesión docente, así como contribuir a formar a los alumnos-practicantes como profesionales reflexivos y más críticos de su futuro desempeño docente. Es posible lograrlo a través de una permanente reflexión sobre los dilemas del ejercicio docente, de lo contrario estos pueden orientar su desarrollo profesional hacia una racionalidad excesivamente técnica que prescinde de la reflexión sobre la acción. Finalmente, ello derivará en el cumplimiento de la función de aprendizaje constante en la enseñanza, excediendo la demostración y aplicación de conocimientos y técnicas adquiridos por el estudiante de educación durante el trayecto de su formación.

Las prácticas preprofesionales deben priorizar la autorreflexión y valoración por las acciones realizadas en ambientes de respeto mutuo, estableciendo relaciones de colaboración entre profesores de las instituciones educativas y los alumnos-practicantes, permitiendo su incorporación también en los procesos de investigación al permitirles comprender la multidimensionalidad del proceso de enseñanza-aprendizaje y descubrir la necesidad de convertirse en investigadores de su propia práctica educativa a fin de desarrollarse profesionalmente. Por el desarrollo de las prácticas debe contextualizarse una serie de situaciones de aprendizaje conducentes a la consolidación de competencias y al desarrollo de capacidades de observación, descripción e interpretación de la realidad y problemática educativa, a la que, cuando sea necesario deben introducirse cambios y transformaciones 
que trasciendan el carácter instrumental de la enseñanza, realizando un ejercicio crítico de la docencia e impulsando verdaderas transformaciones en el aula y en el contexto en general.

Para alcanzar estos objetivos es necesario que todos los docentes universitarios, no solo aquellos que se desempeñan como jefes y/o supervisores de prácticas, incorporen en su práctica pedagógica actividades destinadas a favorecer el desarrollo de competencias críticas y reflexivas que puedan utilizar los alumnos-practicantes durante sus prácticas preprofesionales y desde allí en su futura práctica docente. Asimismo, es necesario establecer líneas de acción dirigidas a fortalecer las prácticas preprofesionales en educación que permitan sentar las bases para una cultura de investigación, acción y reflexión enfocada hacia la producción de conocimientos y no simplemente hacia su aplicación. Ello apunta a un trabajo sistemático de reconstrucción de teorías, validación y contraste con experiencias prácticas orientadas a favorecer la formación profesional docente; se trata de concretar nuevas rutas hacia la vinculación práctica teoría-práctica.

\section{PRÁCTICAS PREPROFESIONALES Y REALIDAD EDUCATIVA}

Los alumnos de las prácticas preprofesionales tienden a mostrar más interés por lo que son capaces de hacer frente a las situaciones de aprendizaje que se les proponen, por lo que suelen considerar de vital importancia ciertos conocimientos sobre otros, descartando muy rápidamente aquellos conocimientos que consideran que nunca le serán relevantes. Este apresuramiento de juicios hará que los alumnos-practicantes concluyan erróneamente que todos los contextos escolares son iguales, por tanto, lo que no sirvió en la contingencia educativa tampoco servirá después. Asimismo, Gonzales y Fuentes (2010:55) señalan que los futuros docentes utilizan sus ideas previas como filtro para dar sentido al currículo de formación, tanto en 
sus aprendizajes teóricos formales y su posibilidad de traslado a la práctica como en la valoración de la utilidad en las experiencias de campo. Entre las creencias más arraigadas está aquella que señala que enseñar es fácil, que prepararse para enseñar consiste en aprender cómo hacer las cosas (organizar juegos, realizar actividades) y que se aprende de la experiencia.

A esto hay que añadir que durante las prácticas preprofesionales el alumno-practicante se encuentra bajo la presión de las múltiples y simultáneas demandas del docente jefe de prácticas y con el grupo de estudiantes con los que le corresponde actuar y otros. Ante estos requerimientos activa su repertorio intelectual, emocional y a partir de él actúa en el contexto escolar, que al mismo tiempo le plantea sus contradicciones y aciertos. De allí la relevancia de atender en el proceso de formación no sólo los elementos conceptuales, procedimentales, sino además los actitudinales, afectivos y emocionales.

También se ha podido constatar que algunos practicantes elaboran previamente modelos docentes que no se corresponden con la realidad, haciendo que el proceso de «enseñar» y «aprender a enseñar» se torne aún más complejo y sea más difícil de asumir, especialmente, si se carece de un adecuado acompañamiento de un jefe de prácticas involucrado en el proceso, provocando que estos sufran una inicial desilusión de lo que significa ser docentes. La práctica preprofesional permite a los futuros docentes tomar consciencia de que aquello que es relevante en un contexto escolar, no lo es necesariamente para otro.

Escobar (2007:191-192) describe una serie de problemas expresados por los estudiantes durante sus prácticas preprofesionales en relación a su desempeño, estos refieren que tienen dificultades para llegar a los centros de prácticas (tanto por la distancia y/o por la dificultad para integrarla en el horario laboral de los estudiantes que se encuentran trabajando), lo demandante de los trabajos exigidos por la propia naturaleza 
de la prácticas con el resto de los trabajos académicos solicitados por otras asignaturas y las dificultades para acceder a la documentación y elaborar acciones y diversos tipos de proyectos que son necesarios implementar en las instituciones educativas donde se realizan las prácticas. Al indagar entre docentes supervisores de prácticas acerca del desempeño de los alumnospracticantes indican que estos presentan dificultades para realizar los trabajos solicitados por que presentan problemas de comprensión lectora y redacción, inseguridad ante los grupos de escolares por falta de experiencia, dificultad para trabajar en equipos, desinterés o apatía para involucrarse en la situación de los centros de práctica y en particular de los estudiantes a su cargo.

En estas condiciones es muy importante acompañar al alumnopracticante en la construcción de una experiencia docente donde acepte la diversidad, el diálogo, los contextos inestables, la propia reflexión como motor en la construcción de conocimientos, a la vez que, crea y recrea nuevas lecturas de la realidad educativa desde los conocimientos construidos vivencialmente.

\section{HACIA UNA PRÁCTICA PREPROFESIONAL REFLEXIVA}

En la actualidad es innegable que las permanentes transformaciones de las tecnologías de la información y la comunicación redefinen las características de nuestra sociedad en diversos ámbitos de nuestra interacción social, lo que se hace más evidente cuando constantemente las demandas educativas requieran incorporar nuevas capacidades que posibiliten a los futuros ciudadanos no quedar excluidos en una sociedad donde el acceso a los conocimientos e información está asociado al manejo de saberes digitales. $\mathrm{Si}$, para que los estudiantes, futuros docentes, sean capaces de atender estos 
requerimientos requieren adquirir conocimientos y capacidades específicas, los estudiantes de ciencias de la educación como futuros educadores deben prepararse para un ejercicio docente con una práctica reflexiva, estimuladora de la innovación y la cooperación. Siendo las prácticas preprofesionales el espacio donde estos deben desarrollar las competencias reflexivas y críticas que conformen esquemas mentales con los cuales el futuro docente encuentre un asidero para interpretar e intervenir la realidad educativa.

La reflexión como un proceso interno, se puede producir mediante una reflexión sobre la acción o en la acción, que en algunos casos puede plantearse como un proceso individual, pero que indudablemente debe ser considerada una acción colectiva y contextual. Este proceso de reflexión requiere ser aprendido y ejercitado para que se constituya en una actitud y habilidad permanente, siendo la práctica preprofesional un espacio propicio para iniciar en la práctica reflexiva a los futuros docentes, articulando la teoría y la práctica, aproximando, gradualmente, a los alumnos-practicantes al trabajo profesional.

La práctica reflexiva es una auto-inspección de las actitudes personales, creencias, valores y comportamientos propios de los docentes y en la práctica preprofesional, de los alumnos-practicantes. Estas son habilidades vitales para los facilitadores de cambio, como una forma de ser más concientes en nuestro propio actuar, poder y eficiencia. La práctica reflexiva no solo incluye un análisis crítico de manera particular de ver el mundo, sino también de la forma de actuar en este: formas de saber y ser que cada futuro docente lleva en su persona, emociones, expresiones artísticas o acciones prácticas, así como su propio razonamiento cognitivo.

Una docencia reflexiva-analítica supone que las decisiones pedagógicas tomadas por los docentes se realizan en base a una consciente y cuidadosa consideración de los supuestos didácticos-pedagógicos y a la 
evaluación de consecuencias técnicas, educacionales y éticas resultantes de las decisiones tomadas antes, durante y después de sus acciones docentes. Para lo cual el docente debe tener un conocimiento extensivo del contenido que enseña, de las opciones teóricas y pedagógicas, de las características de los estudiantes y de las situaciones que influyen en el aula, escuela y sociedad en la cual trabaja.

Las prácticas preprofesionales deben permitir a los alumnospracticantes elaborar ciertos conocimientos y procedimientos específicos para enfrentar diversos aspectos del proceso de enseñanza-aprendizaje que serán recurrentes y cómo manejar aquellos que surgen de manera imprevista. El profesional de la educación adquiere destreza en sus competencias profesionales en el ejercicio de su práctica docente, siendo la práctica preprofesional el espacio donde empieza a reconocer los conocimientos prácticos-profesionales y constituyen uno de los puntos de partida más sustanciales para la reflexión docente.

Los conocimientos académicos, las experiencias cotidianas y las creencias personales de los docentes se conjugan en el desarrollo de la identidad y de la identidad profesional de los docentes, construyendo una práctica profesional autocrítica reflexiva. Esta reflexividad sobre su propio desempeño como futuro docente es una fuente para nuevas propuestas e innovaciones que, a su vez, deberán seguir siendo repensadas de manera personal y colegiada. Asimismo, esta interacción permanente entre la reflexión y la acción constituye el corazón de un estilo de desarrollo profesional que es capaz de construir y comunicar un saber pedagógico relevante y emergente.

Una práctica preprofesional reflexiva debe propiciar una formación docente que promueva el desarrollo de complejas competencias profesionales y capacidades para la autonomía, la reflexión y la crítica que integren el 
pensamiento y la acción, a través de un proceso donde interactúen teoría y práctica, favoreciendo el desarrollo de la capacidad de comprensión situacional, los juicios razonados, decisiones inteligentes y reflexivas frente a lo dinámico que son las situaciones de aprendizaje que se suceden en las aulas con el ánimo de mediar positivamente en el proceso de enseñanzaaprendizaje de quienes tendrán a cargo la mejora de la calidad educativa, estimulando durante las prácticas preprofesionales la interpretación de las situaciones de aprendizaje como un todo.

Los alumnos-practicantes durante sus prácticas deben adquirir una actitud reflexiva, capaz de comprender la realidad en la que intervienen, desarrollando y construyendo esquemas flexibles de pensamiento y actuación, la investigación y la innovación de propuestas didácticas o pedagógicas que implican la superación de perspectivas instrumentalistas del proceso educativo, que requieren de una mejor comprensión e interpretación de la singularidad de cada situación concreta apoyada en teorías, experiencias previas, pero no limitadas a estas.

\section{LAS PRÁCTICAS PREPROFESIONALESY LA INVESTIGACIÓN- ACCIÓN}

En la investigación-acción confluye y se desarrolla un proceso de andamiaje entre la teoría y la práctica, es decir, entre el conocimiento técnico y teórico que deben manejar los docentes para enseñar a sus estudiantes y el conocimiento tácito que nace de su propia experiencia en el aula. En este sentido, el aprendizaje de los docentes no solo implica un aprendizaje técnico o la utilización de ciertas metodologías asociadas a los diversos contenidos que tratan en las aulas, sino también el reflexionar acerca de su propia práctica educativa. Esta reflexión acerca de su acción pedagógica 
constituye uno de los elementos vitales que debe considerar el docente a la hora de enfrentar y definir su práctica, ya que le otorgará sentido a su quehacer profesional y a lo que realmente enseña y cómo enseña en el aula, cómo es capaz de mediar y construir conjuntamente con sus alumnos el conocimiento.

Es durante el desarrollo de las prácticas preprofesionales que se puede iniciar activamente a los alumnos-practicantes en la necesidad de integrar la investigación-acción en su desempeño docente como parte de su cotidianidad en las aulas y en el entorno educativo. Así desde las prácticas preprofesionales se pueden identificar oportunidades para investigar $y$ proponer mejoras de la enseñanza, en procesos de observación, planificación, acción y revisión de las acciones educativas.

Desde esta perspectiva el conocimiento práctico no es el objetivo de la investigación-acción sino inicio de un proceso de descubrimiento que se transforma en la base del proceso de concientización de la labor realizada por el docente. La concientización es una idea central y una meta en la investigación-acción, tanto en la producción de conocimientos como en las experiencias concretas de acción.

La investigación-acción se centra en la posibilidad de aplicar categorías científicas para la comprensión y mejoramiento de la organización, partiendo del trabajo colaborativo de los propios docentes-investigadores, quienes realizan un proceso continuo en espiral. En este espacio se dan los momentos de problematización, diagnóstico, diseño de una propuesta de cambio, aplicación de la propuesta y evaluación, para luego reiniciar un nuevo circuito partiendo de una nueva problematización.

A través de las prácticas preprofesionales la ejecución de proyectos de investigación-acción permite estimular el espíritu de indagación crítica de los alumnos-practicantes. Se delinean actividades que requieren realizar 
procesos de investigación, reflexión y acción en los espacios educativos, de manera que estos puedan ir perfeccionando su práctica docente mediante el desarrollo de las capacidades de discriminación y de juicio del profesional en situaciones concretas de aprendizaje.

Dado que la investigación-acción pone un acento muy fuerte en la autorreflexión de la propia práctica educativa de aquellos que están inmersos directamente en ella, hace que los docentes reflexionen permanentemente sobre lo que hacen, dicen y sienten durante su práctica. En consecuencia, el docente-investigador se convierte en un permanente indagador activo dentro del contexto de las aulas a su cargo, problematizando su propia práctica y analizando profundamente los aspectos que subyacen en ella.

Una de las funciones esenciales de la investigación-acción consiste en producir conocimientos desde la práctica educativa a través de una autorreflexión sistemática docente, utilizando racionalmente los diferentes métodos y técnicas de recolección de información. En la perspectiva de la investigación-acción el conocimiento es producido no solo para planificar las acciones con fines instrumentales, ni para producir conocimientos; si no con la finalidad de comprender determinadas situaciones educativas problemáticas, sino que se trata de una producción que apunta a enfrentar dichos problemas que pueden estar obstaculizando el proceso de enseñanzaaprendizaje, restando eficacia y no permiten el logro de los objetivos propuestos en la planificación curricular. Los resultados de la investigación van aportando las ideas necesarias para facilitar el desarrollo de las personas y de las instituciones.

Considerando la importancia del papel del conocimiento para el desarrollo de un país, la investigación-acción permite a las instituciones educativas producir un saber sobre sus propias prácticas educativas que ayuden a mejorar los resultados de su misión institucional. La investigación- 
acción incrementa las posibilidades de mejorar la calidad del quehacer institucional y puede, de esta manera, servir de referente para otras organizaciones y contribuir al desarrollo, a través de espirales de reflexión, en una propuesta de transformación de la práctica que contiene, una vez iniciado el proceso, diversas posibilidades.

\section{EVALUACIÓN DE LA PRÁCTICA PREPROFESIONAL}

García, Laredo y Carranza (2008:6-7) consideran la evaluación educativa, el análisis del pensamiento y la acción docente que debe, ubicarse en el marco de un proyecto institucional en el cual la actividad docente y su perfeccionamiento ocupen un lugar preponderante, siendo necesario contar con un marco de referencia amplio donde es necesario considerar los diferentes momentos de realización de la práctica educativa: planeación, desarrollo y evaluación, concebida como el espacio en el cual se puede confrontar el proyecto institucional, es decir, las metas y políticas institucionales, con las acciones docentes propiamente dichas.

Sin embargo, la mayoría de las instituciones de formación docente tratan de replicar los modelos tradicionales de formación docente haciendo que para los alumnos-practicantes sea una experiencia poco flexible, que tiende a homogenizar los programas de estudio, recargados de contenidos y donde la experiencia práctica se orienta a la elaboración de productos preestablecidos y no valoran los contextos socio-educativos. Siendo necesario replantear las maneras como se están llevando a cabo la evaluación de las prácticas preprofesionales y reflexionar en las competencias generales y específicas que debe poseer el futuro docente, las mismas que están declaradas explícitamente en el perfil y los planes curriculares de formación profesional de la Carrera Profesional de Educación. 
Desde esta apreciación la evaluación de las prácticas preprofesionales deben partir del trabajo reflexivo de los alumnos-practicantes, de su acción didáctica y pedagógica así como su capacidad para proponer mejoras en los procesos de enseńanza y de aprendizaje, lo que conlleva a plantear una evaluación de las prácticas preprofesionales no sólo en los aspectos operativos y comportamentales, sino también para establecer el nivel de comprensión del ejercicio docente realizado por los alumnos-practicantes y las ideas que desarrollan de sí mismos.

La evaluación de las prácticas preprofesionales requiere de una permanente supervisión. Debe registrar y verificar las evidencias de producción y los servicios realizados por los alumnos-practicantes. Se espera que sean muestra de sus capacidades y habilidades cognoscitivas, laborales y productivas, y que permitan precisar el grado alcanzado de eficacia y eficiencia docente.

En la evaluación de las prácticas preprofesionales se debe privilegiar un paradigma de la comprensión, la reflexión y la mejora del proceso de $\underset{\infty}{\infty}$ enseñanza-aprendizaje. Las características de la evaluación entendida desde dicho paradigma toman en cuenta el contexto y los procesos, y no únicamente los resultados alcanzados por los alumnos-practicantes en condiciones de libertad para usar métodos diversos y suficientemente sensibles para captar la complejidad de los fenómenos educativos a los que se enfrentan; estando más atentos a los valores que a los indicadores técnicos; no convirtiendo la complejidad en simplificación; recordando permanentemente que el proceso educativo tiene un carácter estructural y holístico; privilegiando el diálogo, la participación y la reflexión.

En este sentido las escalas de apreciación o descriptores de desempeño de los alumnos-practicantes en las diversas situaciones de práctica educativa deben dimensionar adecuadamente el alcance de comprensión de la realidad 
educativa siendo necesario que las prácticas preprofesionales permitan verificar si los futuros docentes han logrado:

- Posibilitar el desarrollo de aprendizajes flexibles, significativos y con sentido, asumiendo un papel de facilitador del conocimiento y haciendo que sean los alumnos los actores activos de su proceso aprendizaje.

- Favorecer y desarrollar el deseo de aprender, impulsando el compromiso hacia el aprendizaje de sus futuros alumnos.

- Integrar en su práctica docente los aspectos didácticos-pedagógicos con una visión ética y moral que procure una visión integral del proceso de aprendizaje de sus alumnos.

- Integrar la teoría y práctica de las ciencias de la educación, priorizando el tipo de aprendizajes que se desea construir sobre los aprendizajes que solo se quieren transmitir.

Es evidente que en la evaluación de las prácticas preprofesionales se debe hacer prevalecer lo pedagógico sobre lo administrativo, enfatizando en la necesidad de que los alumnos-practicantes otorguen la debida importancia al contexto socio-cultural de sus estudiantes y de las instituciones educativas. Todo ello con el fin de seleccionar las mejores estrategias, y recursos pedagógicos y didácticos que den respuesta a sus necesidades educativas; las mismas que pueden ser atendidas en el marco de los objetivos de aprendizajes expresados en el P.E.I., y en la planificación curricular. Incluso, de ser necesario, se puede hacer a través del diseño e implementación de proyección de innovación pedagógica. 
Consideramos que las Prácticas Preprofesionales en Educación deben constituir el espacio donde los alumnos-practicantes, próximos a incorporarse a la práctica docente, deben desarrollarse de manera integral. Desde este presupueste, las prácticas permiten alcanzar un dominio eficiente de la planificación y ejecución de los diseños pedagógicos en el aula y en las instituciones educativas; analizar los contenidos curriculares y su contextualización en sus respectivas áreas curriculares, la cual responda a la necesidades de los alumnos y a la comunidad a la que pertenece la institución educativa; desarrollar una visión holística del proceso de aprendizaje que tome en cuenta aspectos cualitativos de su propio accionar pedagógico y el aprendizaje de sus futuros estudiantes, implementando y propiciando el desarrollo de las capacidades de investigación de situaciones concretas que son factibles a través de la investigación-acción. Así estaremos seguros de que los docentes formados de esta manera, verdaderamente, tendrán las competencias, recursos e identidad que los comprometa activamente con el mejoramiento de la calidad del sistema educativo en nuestro país. 


\section{BIBLIOGRAFÍA}

EsCOBAR, Nancy

2007 «La práctica profesional docente desde la perspectiva de los estudiantes practicantes y tutores». Acción Pedagógica, Instituto de Mejoramiento Profesional del Magisterio, N. 16, enero-diciembre, pp. 182-193. Caracas.

García Cabrero Cabrero, Benilde; Javier Laredo Enriquez y Guadalupe Carranza PeÑA

2008 «Análisis de la práctica educativa de los docentes:pensamiento, interacción y reflexión». Revista electrónica de investigación educativa $<$ http://redie.uabc.mx/NumEsp1/contenido-garcialoredocarranza. html>. Consulta hecha en 01/06/2012.

González Sanmamed, Mercedes y Eduardo José Fuentes Abeledo 2011 «El Practicum en el aprendizaje de la profesión docente». Revista de educación, Instituto Nacional de Evaluación Educativa Ministerio de Educación, Cultura y Deporte, N. 354, abril, pp. 47-70. Madrid.

SAYAgo, Zoraida Beatriz y María Auxiliadora CHAcón

2006 «Las prácticas profesionales en la formación docente. Hacia un nuevo diario de ruta». EDUCERE - La revista venezolana de educación, Universidad de los Andes, año 10, N. 32, enero-febrero, pp. 55-66. Mérida. 
Sime P, Luis

2004 «Rutas para el desarrollo profesional docente». Educación, revista del departamento de Educación de la PUCP, Vol. XIII, N. 25, setiembre, pp. 61-75. Disponible en Blog PUCP. <http://blog. pucp.edu.pe/media/624/20080121-Rutas\%20para\%20el\%20 desarrollo\%20profesional\%20docente.pdf>. Consulta hecha en $01 / 06 / 2012$. 